When thought suppression backfires: its moderator effect on eating psychopathology.

\author{
Cláudia Ferreira, $\mathrm{PhD}^{* 1}$ \\ Lara Palmeira, MSc* \\ Inês A. Trindade, $\mathrm{MSc}^{*}$ \\ Francisca Catarino, $\mathrm{MSc}^{*}$
}

* Cognitive and Behavioural Research Centre, University of Coimbra

${ }^{1}$ Correspondence concerning this manuscript should be addressed to:

Cláudia Ferreira

CINEICC, Faculdade de Psicologia e Ciências da Educação, Universidade de Coimbra Rua do Colégio Novo, Apartado 6153

3001-802 Coimbra, Portugal

Telefone: (+351) 239851450

Fax: (+351) 239851462

Email: claudiaferreira@,fpce.uc.pt 


\title{
When thought suppression backfires: its moderator effect on eating
}

\section{psychopathology.}

\begin{abstract}
Recently, several studies point out the importance of thought suppression as a form of experiential avoidance in different psychopathological conditions. Thought suppression may be conceptualized as an attempt to decrease or eliminate unwanted thoughts. However, it encloses a paradoxical nature, making those thoughts hyper accessible and placing an extra burden on individuals. This avoidance process has been associated with several psychopathological conditions. However, its role in eating psychopathology remains unclear.
\end{abstract}

The present study aims to explore the moderation effect of thought suppression on the associations between unfavourable social comparison through physical appearance, body image dissatisfaction and eating psychopathology severity in a sample of 211 female students.

Correlational analysis showed that thought suppression is associated with psychological inflexibility and eating disorders' main risk factors and symptoms. Moreover, two independent analyses revealed that thought suppression moderates, as it amplifies the impact of unfavourable social comparisons through physical appearance (model 1) and body image dissatisfaction (model 2) on disordered eating attitudes and behaviors. Hence, for the same level of these body-related internal experiences, those women who present higher levels of thought suppression present higher eating psychopathology. Taken together, these findings highlight the key role of thought suppression in eating psychopathology and present important clinical implications.

Key-words: thought suppression; eating psychopathology; social comparisons; body image dissatisfaction. 


\section{Introduction}

Eating disorders are widely recognized as complex and multidetermined psychopathological conditions (Stice, 2001). Literature has been highlighting the important role of body image dissatisfaction as a major risk factor in the etiology and development of eating psychopathology (e.g., Stice, Marti, \& Durant, 2011; PintoGouveia, Ferreira, \& Duarte, 2012). In fact, the perception of a significant discrepancy between the actual body image and the desired one (i.e., body image dissatisfaction) has been linked to several disordered attitudes and behaviors that aim to weight and shape control (e.g., dieting; Stice et al., 2011). Additionally, several studies have pointed out that the perception of this discrepancy may be experienced as a social threat, putting one at risk of being ignored, criticized or rejected by others (Burkle, Ryckman, Gold, Thornton, \& Audesse, 1999; Ferreira, Pinto-Gouveia, \& Duarte, 2013b).

In fact, physical appearance is a self-evaluative central domain for the majority of women, as it is intrinsically linked not only to beauty standards, but also to positive psychological attributes (e.g., dominance, perseverance and social competences), health, success and happiness (Kanazawa \& Kovar, 2004; Sypeck et al., 2006; Webster \& Driskell, 1983). Furthermore, in Western societies, presenting a thin body image (similar to the bodies presented by models or celebrities) is considered ideal as it offers several social advantages, such as positive social attention, approval and favorable appreciation from others (Ferreira, Pinto-Gouveia, \& Duarte, 2013a; Gilbert, 2002; Troop, Allan, Treasure, \& Katzman, 2003). These social advantages define a favorable and secure social rank (Barkow, 1980). In contrast, presenting a physical appearance different from the valued ones (e.g., overweight) frequently triggers unfavorable social comparisons, which in turn lead to feelings of inferiority, inadequacy and undesirability (e.g., Puhl \& Heuer, 2009). Moreover, several studies demonstrate the central role of 
these feelings of inferiority and inadequacy on the development of eating psychopathology (Pinto-Gouveia et al., 2012; Troop et al., 2003).

Nevertheless, the impact of these well known risk factors (body image dissatisfaction and unfavorable social comparisons) in disordered eating attitudes and behaviors may be enhanced by maladaptive psychological processes.

Thought suppression is considered one of those maladaptive psychological processes and it relates to the attempt to diminish or eradicate unwanted thoughts by trying not to think about a specific topic. However, trying to suppress a thought has detrimental side effects, given that this process holds a paradoxical nature, increasing the frequency and impact of thoughts (Salkovskis \& Campbell, 1994; Wegner, Schneider, Carter, \& White, 1987). Meta-analytic studies concerning these paradoxical effects confirm the rebound effect of this ineffective control strategy, showing that suppressed thoughts often return even more strongly (Abramowitz, Tolin, \& Street, 2001; Wegner \& Pennebaker, 1993). Furthermore, thought suppression has been consistently associated with several psychopathological conditions like depression (Wenzlaff \& Bates, 1998), posttraumatic stress disorder (Shipherd \& Beck, 1999) and obsessive-compulsive disorder (Janeck \& Calamari, 1999). Specifically in the eating disorders field, a growing body of evidence has illuminated the importance of thought suppression (e.g., Polivy, 1998; Ward, Bulik, \& Johnston, 1996; Wenzlaff \&Wegner, 2000).

Currently, Western societies are full of readily available palatable foods. It is likely that this intensive exposure to food-related cues has an influence on psychological processes, such as thoughts, emotions and motivations, increasing foodrelated thoughts (Lowe et al., 2009). Moreover, the social pressure to have a thin body induces great efforts to control eating behaviors, attitudes and thoughts, which is 
difficult in this obesogenic environment (Brownell \& Horgen, 2004). In fact, this is especially hard for at-risk groups such as eating disordered, obese, dieters and individuals with dietary restraint attitudes (e.g., Barnes \& Tantleff-Dunn, 2010; Ogden, 2003; O'Connell, Larkin, Mizes, \& Fremouw, 2005; Soetens \& Braet, 2006). For these specific populations, food and eating-related thoughts may function as warning signals (i.e., unwanted internal experiences) increasing control and suppression attempts and preoccupation with food (Herman \& Polivy, 1993). These thought suppression attempts paradoxically make food and eating-related thoughts hyper accessible and may even unsettle individual's eating behaviors (Soetens \& Braet, 2006; Wenzlaff \& Wegner, 2000). In fact, several studies have been consistently showing the association between food-related thought suppression and increased food intake, eating disorder symptoms, food cravings and binge-eating episodes (e.g., Barnes \& Tantleff-Dunn, 2010; Pop, Misclea, \& Hancu, 2004; Ward, Bulik, \& Johnston, 1996).

Although there is empirical evidence on the relevance of thought suppression on disordered eating, its role in this psychopathological process is yet scarcely known. Therefore, the key goal of the present paper is to explore the moderator effect of thought suppression on the relation between two main eating psychopathology's risk factors (body image dissatisfaction and social comparison through physical appearance) and eating psychopathology severity. The hypothesis that underlies this study is that these thought control strategies may amplify the impact of the risk factors on disordered eating attitudes and behaviors.

\section{Materials and methods}

\section{Participants}


Two hundred and eleven female students aged between 15 and 23 years-old participated in this study. They presented a mean age of $18.57(S D=2.02)$ and of 12.29 $(S D=1.85)$ years of education. The average BMI was $21.41(S D=2.77)$.

\section{Procedures}

The current research was presented and approved by the Ethics Committee Boards of all the educational institutions participating in the study. The female students who participated in the study were recruited from several high schools and also from the University of Coimbra. All participants (and their parents, if they were minor) were required to sign a consent form to take part in the study. Participants were fully informed about the aim of the study and the confidential nature of the data before completing several self-report measures during class (approximately 15 minutes) in the presence of the teacher and one of the researchers that that provided further explanations when needed. Students who did not participate were given a task by the teacher.

\section{Measures}

Demographic Data. Participants were asked about their age, years of education, current height and weight. Then $\mathrm{BMI}\left(\mathrm{Kg} / \mathrm{m}^{2}\right)$ was calculated.

White Bear Suppression Inventory (WBSI; Wegner \& Zanakos, 1994; PintoGouveia, \& Albuquerque, 2007). The WBSI is a 15-item scale that assesses thought suppression using several statements that the participant has to score on a 5-point Likert Scale. Higher scores reveal elevated tendencies to suppress unwanted thoughts. The 
scale showed good reliability in both the original study (Cronbach's alphas from.87 to $.89)$ and in the Portuguese validation study.

Social Comparison through Physical Appearance Scale (SCPAS; Ferreira et al., 2013b). This is a self-report scale, with 12 items, that measures women's perceptions of their social standing based on physical appearance. Participants are asked to compare themselves to others based on physical appearance, using a 10-point Likert rating scale with bipolar constructs. It comprises two different parts. In part A, participants are asked to compare themselves to peers and in part $\mathrm{B}$ to compare themselves to models/celebrities. For the purpose of the current study, only part B was considered and the scale was reverse scored in order to obtain a measure of unfavourable social comparisons. Part B showed good internal consistency in the original study $(\alpha=.96)$.

Figure Rating Scale (FRS; Thompson \& Altabe, 1991; Ferreira, 2003). The FRS consists of a series of nine silhouettes of gradually increasing sizes in accordance to their number (1 - thinnest; 9 - largest). The respondents are asked to indicate which of the figures corresponds to their current and desired body shape. One's body image dissatisfaction (BD) is calculated by the discrepancy between these two figures. As shown in the original study, the FRS has adequate temporal, convergent and divergent reliabilities.

Eating Disorder Examination Questionnaire (EDE-Q; Fairburn \& Beglin, 1994; Machado, 2007). Developed through the Eating Disorder Examination interview, the EDE-Q is a self-report measure that assesses eating psychopathology-related attitudes and behaviors in four subscales: dietary restraint, eating concern, shape concern and weight concern. In the present study only the global score of EDE-Q was used. The EDE-Q showed very good levels of internal consistency, temporal reliability, and concurrent and discriminative validities (Fairburn, 2008). 
The Cronbach's alphas of all study variables are presented in Table 1.

\section{Data analysis}

Data analyses were performed using IBM SPSS Statistics 20 (IBM Corp, 2011).

To explore the association between all study variables (thought suppression, psychological inflexibility, general social comparison, social comparison through physical appearance_models, body image dissatisfaction and the severity of eating disorders symptoms, Pearson correlation coefficients were performed (Cohen, Cohen, West, \& Aiken, 2003).

Two different models were conducted to explore the moderator effect of thought suppression. In the first analysis, the moderator effect of thought suppression (WBSI) on the relationship between social comparison through physical appearance_models (SCPAS_Models) and eating psychopathology (EDE-Q) was examined. Then, in a second analysis, the moderator effect of thought suppression on the relationship between body image dissatisfaction (BD) and eating psychopathology (EDE-Q) was explored. In these analyses the interaction of a continuous predictor was considered (Cohen, Cohen, West, \& Aiken, 2003). A standardized procedure was used to reduce the error associated with multicollinearity. Thus, the values of the predictors (SCPAS_Models, BD) and the moderator (WBSI) were centered and the interaction product was obtained by multiplying the created variables (Aiken \& West, 1991). Finally, two graphics were plotted (Jose, 2013) considering one curve for each of the three levels of the moderator (low, medium and high) to better understand the relationship between the independent variables (SCPAS_Models, BD) and eating psychopathology with different levels of the moderator variable (WBSI). As recommended by Cohen and colleagues (2003) and since there were no theoretical cut 
points for the moderator variable on the $\mathrm{x}$ axis, in the graphical representations, the three curves were plotted considering the following cut point values: one standard deviation below the mean, the mean and one standard deviation above the mean (Jose, 2013).

\section{Preliminary Data Analyses}

Firstly, the data suitability for the regression analyses was evaluated. Skewness and kurtosis values did not show a serious bias to normal distribution ( $\mathrm{SK}<|3|$ and $\mathrm{Ku}$ $<|8-10|$; Kline, 2005) and multicollinearity was not identified as all variables presented VIF values $<5$, which indicate the absence of $\beta$ estimation problems. Additionally, independence of errors was analyzed through graphic analysis and the value of DurbinWatson (values ranged between 1.622 and 1.739). Overall, these data are adequate for regression analyses.

\section{Results}

Descriptives

Means and standard deviations for the study variables are presented in Table 1.

\section{Correlations}

Pearson product-moment correlations between studied variables are presented in Table 1. Results showed that thought suppression is positively and moderately associated with unfavorable social comparisons based on physical appearance (SCPAS_Models) and eating psychopathy severity (EDE-Q global score). It also presented a positive association, albeit weak, with body dissatisfaction. In turn, no 
significant correlation was found between thought suppression and BMI. Moreover, a partial correlation analysis was performed controlling for age. Results showed the same pattern and magnitude of the association between all study's variables. Therefore, age was not considered in further analysis.

[Table 1 about here]

\section{Moderation analyses}

Given the previous findings and to further understand the role of thought suppression on eating psychopathology severity, two moderator analyses were conducted. It was explored whether thought suppression (WBSI) moderated the relationship of unfavourable social comparisons based on physical appearance (SCPAS_Models; Model 1) and body image dissatisfaction (BD; Model 2) with disordered eating symptoms (EDE-Q).

1.The moderator effect of thought suppression on the relationship between unfavourable social comparisons based on physical appearance and eating psychopathology

Firstly, SCPAS_Models was entered as a predictor in the first step of the regression model (Table 2). On the next step, WBSI was further included as a predictor variable. In both steps of the regression analysis, statistically significant models were obtained [Step 1: $R^{2}=.18, F_{(1,209)}=46.17, p<.001 ;$ Step $2: R^{2}=.27, F_{(2,208)}=38.26, p$ $<.001]$. In the last step, the interaction terms were entered and the model accounted for $31 \%$ of the severity of eating psychopathology variance [Step 3: $F_{(3,207)}=31.50 ; p<$ 
.001]. The regression coefficients analysis showed that the interaction between these two variables was significant $[\beta=.22 ; t=3.66 ; p<.001]$. These results suggest the existence of a moderator effect of thought suppression on the association between social rank based on physical appearance and eating psychopathology symptoms.

the interaction variable was entered and

[Table 2 about here]

2. The moderator effect of thought suppression on the relationship between body image dissatisfaction and eating psychopathology

In the second model (Table 2) the same procedure was performed to explore the relationship between body image dissatisfaction (BD) and eating psychopathology moderated by thought suppression (WBSI). BD was entered in step one as a predictor and in step two WBSI was added [Step 1: $R^{2}=.37, F_{(1,209)}=120.66, p<.001$; Step 2: $\left.R^{2}=.45, F_{(2,208)}=83.83, p<.001\right]$. In the third step, the interaction variable was entered and the model accounted for $49 \%$ of the severity of eating psychopathology variance $\left(F_{(3,207)}=65.77, p<.001\right)$. The obtained regression coefficients results revealed that the interaction of the two variables was significant $(\beta=.21 ; \mathrm{t}=4.11, p<.001)$. Hence, a moderator effect of thought suppression on the relation between body image dissatisfaction and eating psychopathology symptoms was found.

In both moderators analyses, when the interaction variables were entered on the regression models there was a significant increase in $R^{2}$. In addition, they also presented an expressive and significant effect on the severity of eating psychopathology. Therefore, interaction effects between thought suppression and social rank perceptions 
based on physical appearance and body image dissatisfaction were confirmed, suggesting that thought suppression moderates the effect of these risk factors on eating psychopathology severity.

Two graphics were plotted to better understand the relation between these risk factors (SCPAS_Models and BD) and eating psychopathology severity with different levels of WBSI (Figure 1 for SCPAS_Models; Figure 2 for BD) considering one curve for each of the three WBSI levels (low, medium and high).

[Figure 1 and Figure 2 about here]

The graphic representation of model 1 (Figure 1) revealed that, of the individuals who present unfavorable social comparisons based on physical appearance, those who present higher tendency to suppress thoughts (higher scores on WBSI) tend to present higher levels of eating psychopathology in comparison to those with medium and low scores on WBSI. Also, the same pattern was found for those individuals who present more favorable social comparisons based on physical appearance. Nevertheless, in this case, the moderator effect of WBSI on the severity of eating psychopathology was weaker in comparison to the observed effect on medium to high values of SCPAS_Models.

As shown in Figure 2, for the same level of body image dissatisfaction, those participants who presented higher tendency to though suppression (higher WBSI scores) tend to show more severity of eating psychopathology symptoms. From the graphic representation it is possible to observe that the moderator effect of WBSI on the prediction of EDE-Q is stronger when dissatisfaction with body image is more intense. 


\section{Discussion}

The present study highlights the importance of thought suppression on eating psychopathology, corroborating and adding to previous research (e.g., Barnes \& Tantleff-Dunn, 2010; Ward, Bulik, \& Johnston, 1996; Wenzlaff \&Wegner, 2000).

Results showed that thought suppression was linked to the main risk factors for eating disorders (e.g., body image dissatisfaction and unfavorable social comparisons) and with eating psychopathology severity. These results support previous findings which revealed that thought suppression is associated with food cravings, binge-eating episodes and overall eating disorder symptoms (e.g., Barnes, Masheb, White, \& Grilo, 2013; Barnes \& Tantleff-Dunn 2010; Pop, Misclea, \& Hancu, 2004).

Furthermore, the moderator effect of thought suppression on the relationship between social comparisons through physical appearance and body image dissatisfaction and eating psychopathology was explored. Results indicated that the interaction between thought suppression and these risk factors presented a strong and significant effect on overall levels of eating psychopathology severity. Concerning social comparison through physical appearance, our results suggest that for the same level of social comparison, those women who present higher levels of thought suppression reveal higher eating disordered symptoms. Also, the graphic representation of this moderation analysis indicates that the damaging effect of thought suppression is more stressed when women compare themselves more negatively. The same pattern was found for body image dissatisfaction. Indeed, for the same levels of body image dissatisfaction those women who have higher thought suppression levels display higher eating psychopathology severity in comparison to those who have lower levels of thought suppression. Interestingly, the impact of this interaction is more striking for 
women that had higher levels of body image dissatisfaction. This suggests that thought suppression moderates (as it amplifies) the impact of these risk factors on disordered eating attitudes and behaviors.

Taken together, our findings suggest that higher levels of body image dissatisfaction and more unfavorable social comparisons tend to be associated with an increased use of thought suppression, i.e., engagement in more attempts to diminish or eliminate undesirable thoughts. However, this control strategy is ineffective and paradoxical as it may amplify the impact of body image dissatisfaction and unfavorable social comparison on eating psychopathology. Such results can be understood through the occurrence of a rebound effect of thought suppression, which increases the frequency, intensity and impact of the unwanted internal experiences (Abramowitz et al., 2001; Salkovskis \& Campbell, 1994; Wegner et al., 1987). This effect makes it harder to deal with these negative internal events and may even increase the tendency to suppress and control them. Thus, it is possible that women become caught up in this vicious circle, in which body image dissatisfaction and unfavorable social comparisons are fueled by thought suppression, enhancing food, weight and body shape concerns and disordered eating behaviors.

As far as we know, this is the first study to explore the moderator effect of thought suppression on eating psychopathology. In fact, this is a key finding as it entails important future research and clinical implications. Firstly, our data clarifies the crucial role of thought suppression in the maintenance of the psychopathological process that characterizes the disordered eating behavior. Secondly, it points to the importance of addressing thought suppression in patients with eating disorders. This is particularly relevant as it is not possible to prevent experiences of negative social comparison or body image dissatisfaction. Therefore, clinical work should focus on the replacement of 
control strategies to deal with unwanted body-related internal experiences by the development of more adaptive ones (e.g., acceptance, defusion and mindfulness).

Nevertheless, these results entail some methodological limitations. Firstly, the cross-sectional nature of data limits causal conclusions that can be drawn from our findings. Longitudinal studies are needed to determine the directionality of the relations and to corroborate the moderation effect of thought suppression. Secondly, the use of self-reported measures may compromise the generalization of the data. Moreover, we recognize that these models present some limitations since eating psychopathology has a multidetermined and complex nature and other risk factors and emotional regulation processes may be involved. Finally, we chose to use a young female non-clinical sample as it resembles the age and sex risk features to eating disordered attitudes and behaviours (Luce, Crowther, \& Pole, 2008; O'Dea \& Abraham, 2002). Nevertheless, future research should replicate these findings in clinical samples.

In conclusion, the current study adds to previous knowledge as it discloses the pervasive and amplifying effect of thought suppression on the relation between eating psychopathology's main risk factors and disordered eating attitudes and behaviors. 


\section{References}

Abramowitz, J., Tolin, D., \& Street, G. (2001). Paradoxical effects of thought suppression: A meta-analysis of controlled studies. Clinical Psychology Review, $21,683-703$.

Aiken, L. \& West, S. (1991). Multiple regression: Testing and interpreting interactions. Thousand Oaks: Sage Publications.

Allan, S., \& Gilbert, P. (1995). A social comparison scale: Psychometric properties and relationship to psychopathology. Personality and Individual Differences, 19 (3), 293-299. doi:10.1016/0191-8869(95)00086-L

Barkow, J. (1980). Prestige and self-esteem: A biosocial interpretation. In D.R. Omark, F.F. Strayer, \& D.G. Freedman (Eds.), Dominance relations: An ethological view of human conflict and social interaction (pp. 319-332). New York: Garland STPM Press.

Barnes, R.D. \& Tantleff-Dunn, S. (2010). Food for thought: Examining the relationship between food thought suppression and weight-related outcomes. Eating Behaviors, 11, 175-179.

Bond, F.W., Hayes, S.C., Baer, R.A., Carpenter, K.M., Guenole, N., Orcutt, H.K., Waltz, T., \& Zettle, R. (2011). Preliminary Psychometric Properties of the Acceptance and Action Questionnaire-II: A Revised Measure of Psychological Inflexibility and Experiential Avoidance. Behavior Therapy, 42, 676-688. doi: 10.1016/j.beth.2011.03.007

Brownell, K.D., \& Horgen, K.B. (2004). Food fight: The inside story of the food industry, America's obesity crisis, and what we can do about it. New York: McGraw-Hill. 
Burkle, M.A., Ryckman, R.M., Gold, J.A., Thornton, B., \& Audesse, R.J. (1999). Forms of competitive attitude and achievement orientation in relation to disordered eating. Sex Roles, 40, 853-870.

Cohen, J., Cohen, P., West, S., \& Aiken, L. (2003). Applied multiple regression/correlation analysis for the behavioural sciences (3th ed.). New Jersey: Lawrence Erlbaum Associates.

Fairburn, C.G., \& Beglin, S.J. (1994). Assessment of eating disorders: Interview or self-report questionnaire? International Journal of Eating Disorders, 16, 363370.

Ferreira, C. (2003). Anorexia Nervosa: A expressão visível do invisível. Contributos para a avaliação de atitudes e comportamentos em relação ao peso e à imagem corporal [Anorexia Nervosa: The visible expression of the invisible. Contributions for the assessment of attitudes and behaviors in relation to weight and body image]. (Unpublished master's thesis). University of Coimbra, Coimbra.

Ferreira, C., Pinto-Gouveia, J., \& Duarte, C. (2013a). Drive for thinness as a women's strategy to avoid inferiority. International Journal of Psychology \& Psychological Therapy, 13(1), 15-29.

Ferreira, C., Pinto-Gouveia, J., \& Duarte, C. (2013b). Physical appearance as a measure of social ranking: The role of a new scale to understand the relationship between weight and dieting. Clinical Psychology \& Psychotherapy, 20, 55-66. doi:10.1002/cpp.769

Gato, J. (2003). Evolução e ansiedade social [Evolution and social anxiety]. (Unpublished master's thesis). University of Coimbra, Coimbra. 
Gilbert, P. (2002). Body shame: A biopsychosocial conceptualisation and overview, with treatment implications. In P. Gilbert \& J. Miles (Eds.), Body shame: Conceptualisation, research and treatment (pp. 3-54). London: Brunner.

Hayes, S.C., Luoma, J. B., Bond, F., Masuda, A., \& Lillis, J. (2006). Acceptance and Commitment Therapy: Model, processes and outcomes. Behaviour Research and Therapy, 44, 1-25.

Hayes, S.C., Wilson, K.W., Gifford, E.V., Follette, V.M., \& Strosahl, K. (1996). Experiential avoidance and behavioral disorders: A functional dimensional approach to diagnosis and treatment. Journal of Consulting and Clinical Psychology, 64(6), 1152-1168.

Herman, C. P., \& Polivy, J. (1993). Mental control of eating: Excitatory and inhibitory food thoughts. In D.M. Wegner \& J. W. Pennebaker (Eds.), Handbook of mental control (pp.491-505). Englewood Cliffs, NJ: Prentice Hall.

Janeck, A. S., \& Calamari, J. E. (1999). Thought suppression in obsessive-compulsive disorder. Cognitive Therapy and Research, 23, 497-509.

Kanazawa, S. \& Kovar, J.L. (2004). Why beautiful people are more intelligent. Intelligence, 32, 227-243.

Lowe, M.R., Butryn ,M L., Didie, E.R., Annunziato, R.A., Thomas, G.J., Crerand, C.E., Ochner, C.N., Coletta, M.C., Bellace, D., Wallaert, M., \& Halford, J. (2009). The Power of Food Scale: A new measure of the psychological influence of the food environment. Appetite, 53, 114-118.

Machado, P. P. P. (2007). Versão Portuguesa do EDE-Q, 5ª edição [Portuguese Version of the EDE-Q, 5th edition]. Unpublished manuscript. University of Minho. 
Merwin, R.M., Timko, C.A., Moskovich, A.A., Ingle, K.K., Bulik, C.M., \& Zucker, N.L. (2011). Psychological inflexibility and symptom expression in anorexia nervosa. Eating Disorders, 19, 62-82.

O'Connell, C., Larkin, K., Mizes, J. S., \& Fremouw, W. (2005). The impact of caloric preloading on attempts at food and eating-related thought suppression in restrained and unrestrained eaters. International Journal of Eating Disorders, $38,42-48$.

Ogden, J. (2003). What do symptoms mean? British Medical Journal, 327, 409-410.

Pinto-Gouveia, J., \& Albuquerque, P. (2007). Versão portuguesa do inventário de supressão do urso branco. Unpublished manuscprit.

Pinto-Gouveia, J., Ferreira, C., \& Duarte, C. (2012). Thinness in the pursuit for social safeness: An integrative model of social rank mentality to explain eating psychopathology. Clinical Psychology and Psychotherapy. Advance online publication doi: $10.1002 / \mathrm{cpp} .1820$

Pinto-Gouveia, J., Gregório, S., Dinis, A., \& Xavier, A. (2012). Experiential avoidance in clinical and nonclinical samples. International Journal of Psychology and Psychological Therapy, 12(2), 139-156.

Polivy, J. (1998). The effects of behavioral inhibition: integrating internal cues, cognition, behavior, and affect. Psychological Inquiry, 9, 181-204.

Pop, M., Miclea, S., \& Hancu, N. (2004). The role of thought suppression on eatingrelated cognitions and eating pattern. International Journal of Obesity, 28, S222.

Puhl, R. M., \& Heuer, C. A. (2009). The stigma of obesity: A review and update. Obesity, 17(5), 941-964. doi:10.1038/oby.2008.636 
Salkovskis P. M., \& Campbell P. (1994). Thought suppression induces intrusion in naturally occurring negative intrusive thoughts. Behavior Research and Therapy, $32,1-8$

Shipherd, J. C., \& Beck, J. G. (1999). The effects of suppressing trauma-related thoughts in women with rape-related posttraumatic stress disorder. Behaviour Research and Therapy, 37, 99-112.

Soetens, B., \& Braet, C. (2006). 'The weight of a thought': Food-related thought suppression in obese and normal-weight youngsters. Appetite, 46, 309-317. doi:10.1016/j.appet.2006.01.018.

Stice, E. (2001). A prospective test of the dual path-way model of bulimic pathology: Mediating effects of dieting and negative affect. Journal ofAbnormal Psychology, 110, 124-135.

Stice, E., Marti, C. N., \& Durant, S. (2011) Risk factors for onset of eating disorders: Evidence of multiple risk pathways from an 8-year prospective study. Behavior Research and Therapy, 10, 622-627. doi: 10.1016/j.brat.2011.06.009.

Sypeck, M.F., Gray, J.J., Etu, S.F., Ahrens, A.H., Mosimann, J.E., \& Wiseman, CV. (2006). Cultural representations of thinness in women, redux: Playboy magazine's depictions of beauty from 1979 to 1999. Body Image: An International Journal of Research, 3, 229-235.

Thompson, J. K., \& Altabe, M. N. (1991). Psychometric qualities of the figure rating scale. International Journal of Eating Disorders, 10, 615-619. doi: 10.1002/1098- $108 X(199109) 10: 5$

Troop, N. A., Allan, S., Treasure, J. L., \& Katzman, M. (2003). Social comparison and submissive behavior in eating disorders. Psychology and Psychotherapy: Theory, Research and Practice, 76, 237-249. doi:10.1348/147608303322362479 
Ward, T., Bulik, C. M., \& Johnston, L. (1996). Return of the suppressed: Mental control in bulimia nervosa. Behaviour Change, 13, 79-90.

Webster, M., \& Driskell, J. E. (1983). Beauty as status. The American Journal of Sociology, 89, 140-165.

Wegner, D.M., \& Pennebaker, J.W. (1993). Changing our minds: An introduction to mental control. In D. M. Wegner \& J. W. Pennebaker (Eds.), Handbook of mental control (pp. 1-12). Englewood Cliffs, NJ: Prentice-Hall.

Wegner, D.M. \& Zanakos, S. (1994). Chronic thought suppression. Journal of Personality, 62, 615-640.

Wegner, D.M., Schneider, D.J., Carter, S., \& White, T. (1987). Paradoxical effects of thought suppression. Journal of Personality and Social Psychology, 53, 5-13.

Wenzlaff, R. M. \& Bates, D. E. (1998). Unmasking a cognitive vulnerability to depression: How lapses in mental control reveal depressive thinking. Journal of Personality and Social Psychology, 75, 1559-1571.

Wenzlaff, R. M., \& Wegner, D. M. (2000). Thought suppression. In S. T. Fiske (Ed.), Annual review of psychology (Vol. 51, pp. 59-91). Palo Alto, CA: Annual Reviews. 
Table 1

Means $(M)$, standard deviations $(S D)$, cronbach alphas and intercorrelation scores on selfreport measures $(N=255)$

\begin{tabular}{lccccccc}
\hline \multicolumn{1}{c}{ Measures } & $M$ & $S D$ & $\alpha$ & 1 & 2 & 3 & 4 \\
\hline 1. BMI & 21.41 & 2.77 & - & - & - & - & - \\
2. BD & .56 & .91 & - & $.55^{* * *}$ & - & - & - \\
3.SCPAS_M & 75.35 & 17.23 & .97 & $.20^{* *}$ & $.39 * * *$ & - & - \\
4.EDE-Q & 1.33 & 1.22 & .95 & $.39 * * *$ & $.61^{* * *}$ & $.43^{* * *}$ & - \\
5. WBSI & 45.21 & 12.84 & .96 & .13 & $.23 * *$ & $.42^{* * *}$ & $.31^{* * *}$ \\
\hline
\end{tabular}

Note . $\mathrm{BMI}=$ Body Mass Index; $\mathrm{BD}=$ Body Image Dissatisfaction - Figure Rating Scale; SCPAS_M = Unfavourable Social Comparison Trough Physical Appearance Scale_ Models; EDE-Q = Eating Disorder Examination Questionnaire global score; WBSI = White Bear Suppression Inventory

$* * * p<.001$. 
Table 2

Hierarchical multiple regression to analyze WBSI's moderator effect between SCPAS_Models (Model 1) and BD (Model 2) with $E D E-Q(N=255)$

\begin{tabular}{|c|c|c|}
\hline \multirow[b]{2}{*}{ Predictor } & \multicolumn{2}{|c|}{ Eating psychopathology } \\
\hline & $\Delta R^{2}$ & $\beta$ \\
\hline \multicolumn{3}{|l|}{ Model 1} \\
\hline Step 1 & $.18 * * *$ & \\
\hline SCPAS_Models & & $.43 * * *$ \\
\hline Step 2 & $.26 * * *$ & \\
\hline SCPAS_Models & & $.33 * * *$ \\
\hline WBSI & & $.31 * * *$ \\
\hline Step 3 & $.30 * * *$ & \\
\hline SCPAS_Models & & $.29 * * *$ \\
\hline WBSI & & $.33 * * *$ \\
\hline SCPAS_Models xWBSI & & $.22 * * *$ \\
\hline Total $R^{2}$ & $.31 * * *$ & \\
\hline \multicolumn{3}{|l|}{ Model 2} \\
\hline Step 1 & $.36^{* * *}$ & \\
\hline BD & & $.61 * * *$ \\
\hline Step 2 & $.44 * * *$ & \\
\hline BD & & $.54 * * *$ \\
\hline WBSI & & $.29 * * *$ \\
\hline Step 3 & $.48 * * *$ & \\
\hline $\mathrm{BD}$ & & $.51 * * *$ \\
\hline WBSI & & $.30 * * *$ \\
\hline BDxWBSI & & $.21 * * *$ \\
\hline Total $R^{2}$ & $.49 * * *$ & \\
\hline
\end{tabular}




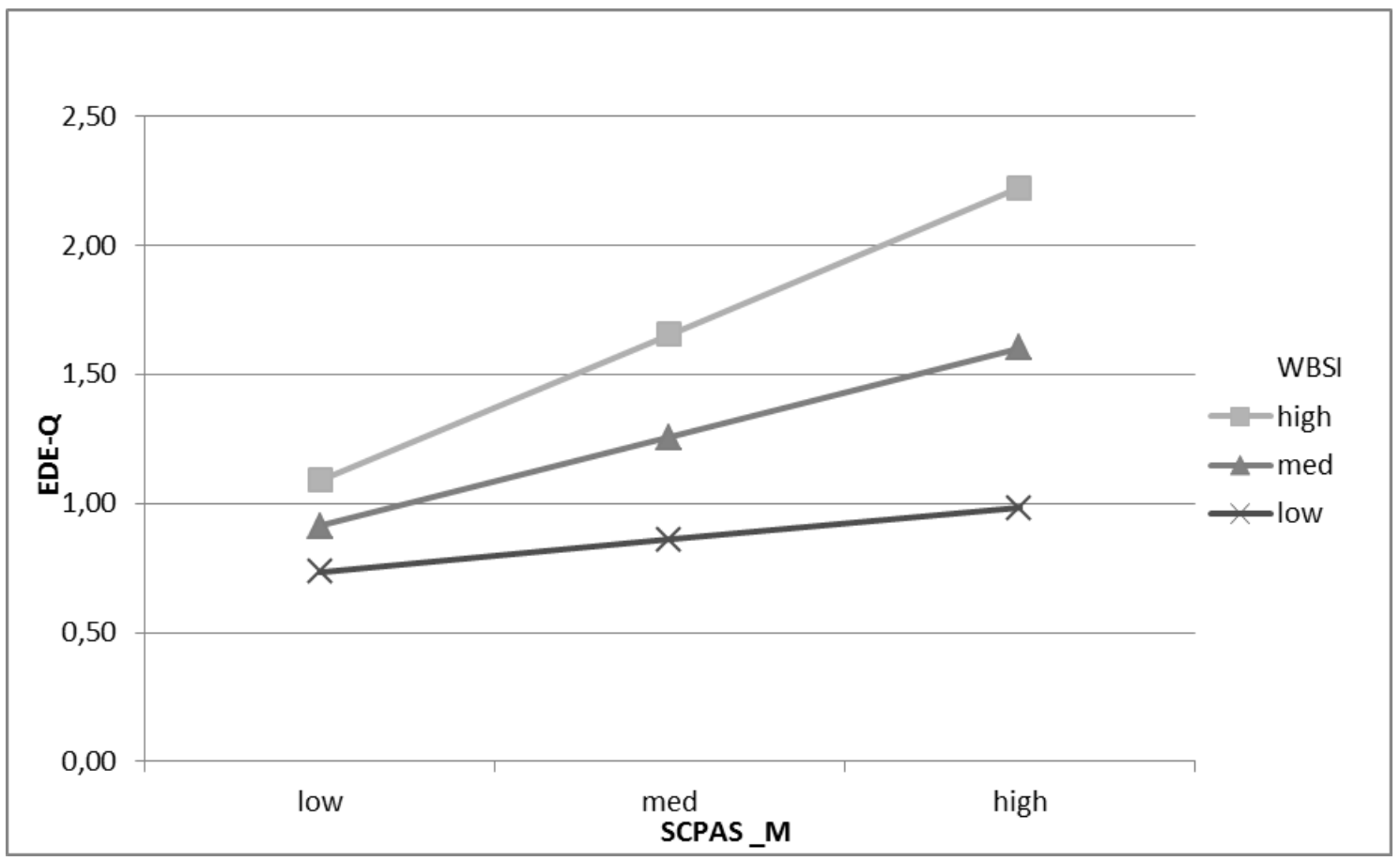

Figure 1. Graphic for the relation between unfavourable social comparisons based on physical appearance (SCPAS_Models) and eating psychopathology (EDE-Q) with different levels of thought suppression (WBSI). 


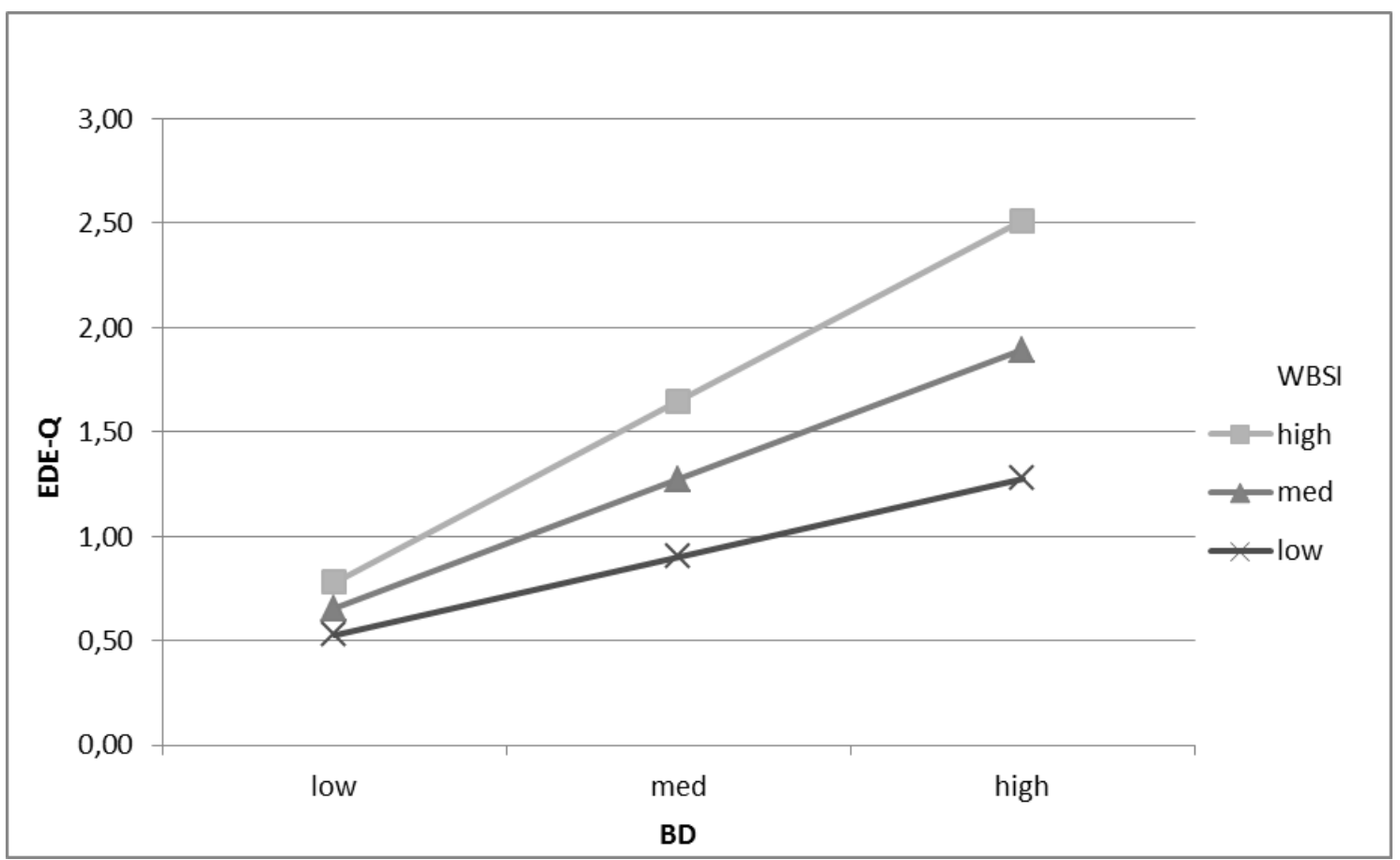

Figure 2. Graphic for the relation between body image dissatisfaction (BD) and eating psychopathology (EDE-Q) with different levels of thought suppression (WBSI). 